\section{VEGF on the brain}

\section{By Tim Fulmer, Senior Writer}

VEGFs are best known for their ability to act on the vasculature and induce angiogenesis. But mounting preclinical evidence suggests VEGF proteins also may act as neurotrophic factors in the CNS, directly influencing the development and survival of neurons in both healthy and diseased states. ${ }^{1,2}$

Researchers at the Katholieke Universiteit Leuven have now shown that VEGF-B may be used to treat amyotrophic lateral sclerosis (ALS). These findings come on the heels of an existing program by the university and partner NeuroNova AB to develop VEGF-A for the same indication. VEGF-B may turn out to be a better candidate for this degenerative disease because of fewer side effects. $^{3}$

The KU Leuven scientists had already shown that intracerebroventricular delivery of recombinant VEGF-A improved motor performance and prolonged survival in a rat model of ALS. ${ }^{4}$ Those results prompted Peter Carmeliet, professor of medicine and director of the Vesalius Research
"We anticipate VEGF-B may in fact have a wider therapeutic window than VEGF-A."

- Peter Carmeliet,

Katholieke Universiteit Leuven blood-brain barrier permeability compared with the effects of cerebrospinal fluid control injection. The same dose of VEGF-B also showed fewer angiogenic side effects than VEGF-A.

As a result, Carmeliet told SciBX, "we anticipate VEGF-B may in fact have a wider therapeutic window than VEGF-A, given that VEGF-B has a reduced potential for unwanted angiogenesis and blood vessel permeability. We will thus continue our preclinical characterization of VEGF-B in motor neuron diseases, looking at higher doses of VEGF-B as well as VEGF-A/VEGF-B combination therapies."

Miriam Mangelus, CEO and cofounder of PhiloGene Inc., said it will be important for additional preclinical safety studies to rule out the potential for VEGF-B to induce blood vessel growth in the brain. "It will probably be necessary to look at long-term dosing effects in preclinical models to be certain that VEGF-B indeed has very little or no angiogenic potential. Even minor vascular side effects could lead to real problems in the CNS," she said.

PhiloGene is developing antiangiogenic variants of VEGF-A to treat age-related macular degeneration (AMD) and solid cancers. The company's strategy takes advantage of the fact that endogenous VEGF-A occurs as multiple isoforms that are either antiangiogenic or proangiogenic. ${ }^{5}$ Thus, delivery of recombinant antiangiogenic VEGF isoforms would be predicted to inhibit vessel growth around tumors and in the eye.

Mangelus told SciBX that PhiloGene's VEGFA splice variants also bind receptors on neurons Center at KU Leuven, and NeuroNova to submit an application to begin a Belgian Phase I trial of sNN0029, a recombinant VEGF-A formulated for intracerebroventricular delivery, to treat ALS.

NeuroNova holds IP from Carmeliet that covers composition of matter, production and medical use of VEGF molecules to treat ALS.

Now, Carmeliet and colleagues at KU Leuven have bolstered the case for another VEGF-B in CNS diseases. In a paper in The Journal of Neuroscience, the group showed that knockout of VEGF-B accelerated onset of motor impairment in a mouse model of ALS compared with that seen in mice that expressed VEGF-B $(p=0.017)$.

In rat models of ALS, intracerebroventricular delivery of recombinant VEGF-B slightly delayed motor degeneration $(p=0.073)$ and significantly prolonged survival $(p=0.016)$ compared with what was seen in rats that received a control injection of artificial cerebrospinal fluid.

Histological analysis revealed that the VEGF-B rats had less loss of neurons in the facial motor nucleus-a group of lower motor neurons-than controls.

The authors proposed that VEGF-B is "a potent and safe therapeutic candidate for the treatment of motor neuron degeneration."

\section{Bringing the B game}

A key result from the Journal of Neuroscience article was that intracerebroventricular delivery of a therapeutic dose of VEGF-B did not significantly increase vascular side effects such as local angiogenesis and and could thus have neuroprotective properties. The company is collaborating with researchers at University of Oxford to study one of the VEGF-A variants in animal models of neuroblastoma, in which the antiangiogenic compound would be expected to show both neuroprotective and antitumor properties.

Sangamo BioSciences Inc. has chosen to upregulate endogenous VEGF-A rather than administer a recombinant form of the factor to treat neurological diseases. "In mice, we originally found that this strategy resulted in angiogenic and neuroprotective effects that are at least comparable to administering [recombinant] VEGF-A but with reduced vascular permeability and hemorrhaging compared with [recombinant] VEGF-A," said Philip Gregory, VP of research. ${ }^{6}$

Sangamo's SB-509, a plasmid DNA encoding a zinc finger DNA-binding protein transcription factor (ZFP TF) to upregulate VEGF-A gene expression, is in Phase II trials to treat diabetic neuropathy and ALS.

Gregory wanted to see more preclinical data on the neuroprotective mechanism of VEGF-B.

"Primary cell-culture studies could provide insight into signaling that occurs in neurons downstream of VEGF-B's interaction with its surface receptor. These data should help us better understand the mechanisms responsible for the molecule's neuroprotective effects," he said.

Xuri Li and colleagues at the NIH's National Eye Institute have found that VEGF-B inhibited apoptosis in mouse models of ocular neurodegenerative disease and stroke. ${ }^{7}$ 


\section{TARGETS \& MECHANISMS}

Li has filed for a U.S. patent covering the use of VEGF-B as an inhibitor of apoptosis in neuronal and other cell types, and the technology is available for exclusive or nonexclusive licensing through the NIH Office of Technology Transfer.

\section{Safely delivering the goods}

Although VEGF-B might represent a promising candidate for neuroprotection, delivery-related hurdles remain.

Susan Croll, associate professor of psychology at Queens College, City University of New York, said two potential challenges facing VEGF$\mathrm{B}$ as a general neuroprotectant "are its inability to cross the blood-brain barrier-a problem for all protein growth factors-and its inability to act directly on neurons that don't express the VEGF receptor 1 , such as neurons in the hippocampus."

Croll and colleagues have shown that direct infusion of exogenous VEGF-A can protect against seizure-induced neuronal cell loss in the rat hippocampus. ${ }^{8}$

Alistair Stewart, VP of commercial research at Allon Therapeutics Inc., suggested that small molecule VEGF-B receptor agonists capable of crossing the blood-brain barrier would be more practical alternatives.

Allon's intranasal AL-108, an eight-amino-acid activity-dependent neuroprotective protein (ADNP), is expected to begin Phase IIb testing to treat Alzheimer's disease (AD) by year end.

"Effective delivery is a key challenge facing any strategy that seeks to treat motor neuron diseases with neurotrophic factors like BDNF [brainderived neurotrophic factor], IGF-1 or VEGF," said Raymond Bartus, EVP and CSO of gene therapy company Ceregene Inc. "To ensure the best chances of clinical success, a therapeutic neurotrophic factor needs to be maintained at a relatively constant level over long periods of time only in a specific class of neurons. Multiple injections of a factor directly into the CNS cannot often meet these requirements owing to the suboptimal diffusion properties of CNS tissue."

Bartus thinks gene therapy is well suited to address these delivery issues. "Here, a single injection of a gene vector encoding the factor is typically sufficient to ensure a long-term therapeutic effect only in the target tissue," he said.

Ceregene's CERE-120, an adeno-associated viral (AAV) type 2 vector encoding neurturin (NTN), is in a Phase II trial to treat advanced Parkinson's disease (PD). CERE-135, an AAV type 2 vector encoding insulin-like growth factor 1 (IGF-1), is in preclinical development to treat ALS. The company hopes to submit an IND to begin a Phase I trial by YE09.

Fred Gage and colleagues at The Salk Institute for Biological Studies have shown that IGF-1 gene therapy targeted to the muscle and subsequently transported to motor neurons delayed disease progression and prolonged survival in a mouse model of ALS. ${ }^{9}$

Carmeliet noted that "delivery of a recombinant protein rather than a gene offers flexible control over dose and duration of administration. However, systemic delivery has generally failed because of the short halflife, immunogenicity and poor blood-brain barrier permeability of the VEGF molecules. For that reason we've developed an intracerebroventricular delivery strategy."

Anders Haegerstrand, CSO of NeuroNova, noted that "direct delivery of VEGF into the brain's fluid-filled ventricles ensures the protein is transported throughout the brain, into the spinal column and ultimately to the higher motor neurons in the neck region. In ALS, degeneration of these neurons can lead to serious problems with swallowing and breathing."

Fulmer, T. SciBX 1(39); doi:10.1038/scibx.2008.939

Published online Oct. 30, 2008

\section{REFERENCES}

1. Zacchigna, S. et al. Nat. Rev. Neurosci. 9, 169-181 (2008)

2. Greenberg, D. \& Jin, K. Nature 438, 954-959 (2005)

3. Poesen, K. et al. J. Neurosci.; published online Oct. 15, 2008; doi:10.1523/JNEUROSCI.1092-08.2008

Contact: Peter Carmeliet, Katholieke Universiteit Leuven, Leuven, Belgium e-mail: peter.carmeliet@med.kuleuven.be

4. Storkebaum, E. et al. Nat. Neurosci. 8, 85-92 (2005)

5. Harper, S. \& Bates, D. Nat. Rev. Cancer 8, 1-8 (2008)

6. Rebar, E. et al. Nat. Med. 8, 1427-1432 (2002)

7. Li, Y. et al. J. Clin. Invest. 118, 913-923 (2008)

8. Nicoletti, J.N. et al. Neuroscience 151, 232-241 (2008)

9. Kaspar, B. et al. Science 301, 839-842 (2003)

\section{COMPANIES AND INSTITUTIONS MENTIONED}

Allon Therapeutics Inc. (TSX:NPC), Vancouver, British Columbia, Canada

Ceregene Inc., San Diego, Calif.

Katholieke Universiteit Leuven, Leuven, Belgium

National Eye Institute, Bethesda, Md.

National Institutes of Health, Bethesda, Md.

NeuroNova AB, Stockholm, Sweden

PhiloGene Inc., Summit, N.J.

Queens College, City University of New York, Flushing, N.Y.

The Salk Institute for Biological Studies, La Jolla, Calif.

Sangamo BioSciences Inc. (NASDAQ:SGMO), Richmond, Calif.

University of Oxford, Oxford, U.K. 